Bull. Korean Math. Soc. 48 (2011), No. 1, pp. 105-114

DOI 10.4134/BKMS.2011.48.1.105

\title{
NORMAL GENERATION OF NONSPECIAL LINE BUNDLES ON ALGEBRAIC CURVES
}

\author{
Seonja Kim $\dagger$ And Young Rock Kim $\ddagger$
}

\begin{abstract}
In this paper, we classify $(C, \mathcal{L})$ such that a smooth curve $C$ of genus $g$ has a nonspecial very ample line bundle $\mathcal{L}$ of $\operatorname{deg} \mathcal{L}=2 g-2-a$ failing to be normally generated, in terms of the number $a$.
\end{abstract}

\section{Introduction}

Let $C$ be a smooth irreducible algebraic curve of genus $g$ over an algebraically closed field of characteristic 0 . A smooth curve $C$ in $\mathbb{P}^{r}$ is said to be projectively normal if the morphism $H^{0}\left(\mathbb{P}^{r}, \mathcal{O}_{\mathbb{P}^{r}}(m)\right) \rightarrow H^{0}\left(C, \mathcal{O}_{C}(m)\right)$ is surjective, i.e., $H^{1}\left(C, \mathcal{I}_{C / \mathbb{P}^{r}}(m)\right)=0$ for each nonnegative integer $m$. We say that a very ample line bundle $\mathcal{L}$ on $C$ is normally generated if the embedded curve $\phi_{\mathcal{L}}(C) \subset$ $\mathbb{P} H^{0}(C, \mathcal{L})$ is projectively normal. It is known that every line bundle $\mathcal{L}$ of $\operatorname{deg} \mathcal{L}>2 g$ is normally generated ([3], [13]), and $C$ admits a very ample line bundle $\mathcal{L}$ of degree $2 g$ failing to be normally generated if and only if $C$ is hyperelliptic ([5]).

We can see that any nonspecial line bundle $\mathcal{L}$ on a general $k$-gonal curve $C$ with $\operatorname{deg} \mathcal{L} \geq 2 g+3-\operatorname{gon}(C)$ is normally generated by Theorem 1 in [5], since $\operatorname{Cliff}(C)=\operatorname{gon}(C)-2$ for a general $k$-gonal curve $([2],[7])$ and so $\operatorname{deg} \mathcal{L} \geq$ $2 g+1-\operatorname{Cliff}(C)+2 h^{1}(C, \mathcal{L})$. For any curve $C$, nonspecial line bundles $\mathcal{L}$ with $\operatorname{deg} \mathcal{L} \geq 2 g+4-\operatorname{gon}(C)$ are normally generated since $\operatorname{Cliff}(C) \geq \operatorname{gon}(C)-3$ by Secant theorem in [4]. Here, $\operatorname{Cliff}(C):=\min \left\{\operatorname{Cliff}(\mathcal{L})=\operatorname{deg} \mathcal{L}-2 h^{0}(C, \mathcal{L})+\right.$ $\left.2 \mid h^{0}(C, \mathcal{L}) \geq 2, h^{1}(C, \mathcal{L}) \geq 2\right\}$ and $\operatorname{gon}(C):=\min \left\{n \mid\right.$ there is a $g_{n}^{1}$ on $\left.C\right\}$.

Received May 18, 2009; Revised September 17, 2009.

2010 Mathematics Subject Classification. 14H45, 14H10, 14C20.

Key words and phrases. algebraic curve, linear series, line bundle, projectively normal, normal generation.

$\dagger$ This work was supported by the Korea Research Foundation Grant funded by the Korean Government (KRF-2008-314-C00011).

$\ddagger$ This research was supported by Basic Science Research Program through the National Research Foundation of Korea(NRF) funded by the Ministry of Education, Science and Technology (331-2008-1-C00012).

The second author appreciated KIAS for warm hospitality when he was an associate member in KIAS 
On the other hand, a general $k$-gonal curve $C$ with $k \leq \frac{g+1}{2}$ has a nonspecial very ample line bundle $\mathcal{L}$ with $\operatorname{deg} \mathcal{L}=2 g+2-\operatorname{gon}(C)$ which fails to be normally generated. In fact, for a general divisor $\sum_{i=1}^{4} P_{i}$ of degree $4, \mathcal{L} \simeq$ $\mathcal{K}-g_{k}^{1}+\sum_{i=1}^{4} P_{i}$ is nonspecial, very ample and not normally generated. Hence we may expect that for a smooth curve having line bundles of degree $\leq 2 g$ which fail to be normally generated is closely related to the existence of some special kinds of linear series.

The purpose of this work is investigating nonspecial very ample line bundles of degree $\leq 2 g$ which fail to be normally generated. In fact, Kato, Keem, and Ohbuchi in [10] determined the conditions for non-special very ample line bundles of degree $2 g-2,2 g-3$ failing to be normally generated. Also the conditions for the cases of degree $2 g-4,2 g-5$ are known in [9]. In this paper we classify such pairs $(C, \mathcal{L})$ with $\operatorname{deg} \mathcal{L}=2 g-2-a>\frac{3 g-3}{2}$ in terms of $a$ that $\mathcal{L}$ is a nonspecial very ample line bundle on $C$ failing to be normally generated. And we obtain a result for the case $\operatorname{deg} \mathcal{L}=2 g-6$ as its corollary and apply this result to the known cases of $2 g-5 \leq \operatorname{deg} \mathcal{L} \leq 2 g-2$ ([9], [10]).

Here we denote $\mathcal{K}_{C}$ by $\mathcal{K}$. Also we use $\mathcal{L}-D$ to $\mathcal{L}(-D)$ and $\mathcal{L}-g_{d}^{n}$ to $\mathcal{L}(-D)$ where $D \in g_{d}^{n}$. For the detailed notations in this paper refer to [1], [6].

\section{Preliminaries}

Let $C$ be a smooth curve of genus $g$ and $\mathcal{L}$ a very ample line bundle on $C$ with $h^{1}(C, \mathcal{L})=s$ and $\operatorname{deg} \mathcal{L}>\frac{3 g-3}{2}+\epsilon$, where $\epsilon=0$ if $\mathcal{L}$ is special, $\epsilon=2$ if $\mathcal{L}$ is nonspecial. Suppose $\mathcal{L}$ is not normally generated, then due to Theorem 3 in [5] there exists a line bundle $\mathcal{A} \simeq \mathcal{L}(-R), R>0$ such that

$$
\operatorname{Cliff}(\mathcal{A}) \leq \operatorname{Cliff}(\mathcal{L}), \operatorname{deg} \mathcal{A} \geq \frac{g-1}{2}, h^{0}(C, \mathcal{A}) \geq 2, h^{1}(C, \mathcal{A}) \geq h^{1}(C, \mathcal{L})+2 .
$$

Note that $\operatorname{Cliff}(C) \leq \operatorname{Cliff}(\mathcal{A}) \leq a-2 s+2$ if $\operatorname{deg} \mathcal{L}=2 g-2-a, a \in \mathbb{Z}_{\geq 0}$. We will frequently use the following.

Setting up: Assume $C$ has a line bundle $\mathcal{L}$ failing to be normally generated with $\operatorname{deg} \mathcal{L}=2 g-2-a>\frac{3 g-3}{2}+\epsilon$. Let $\mathcal{A}, R$ be the same as above. Set $g_{d}^{n}=\left|\mathcal{K} \otimes \mathcal{A}^{-1}\left(-B_{b}\right)\right|$, then $\mathcal{L} \cong \mathcal{K}-g_{d}^{n}-B_{b}+R$, where $B_{b} \in C_{b}$ is the base locus of $\mathcal{K} \otimes \mathcal{A}^{-1}$. Here we may assume that $\operatorname{Supp}\left(B_{b}\right) \cap \operatorname{Supp}(R)=\emptyset$. Let $\phi$ be the morphism associated with $g_{d}^{n}$.

Remark 2.1. If $n=1$, then $\mathcal{L} \simeq \mathcal{K}-g_{m}^{1}-B_{a+4-m}+R_{4}, m \leq a+4, h^{1}(C, \mathcal{L})=0$ by Lemma 2.6 in [9].

For dealing with $n \geq 2$, we need the followings:

Proposition 2.2 (Castelnuovo genus bound, [1]). Let $g_{d}^{r}(r \geq 2)$ be a birationally very ample linear series on a smooth curve of genus $g$. Let $m=\left[\frac{d-1}{r-1}\right]$ and $\epsilon=d-1-m(r-1)$. Then, $g \leq \pi(d, r):=\frac{m(m-1)}{2}(r-1)+m \epsilon$. 
Lemma 2.3. Suppose $\mathcal{L}$ fails to be normally generated with $h^{1}(C, \mathcal{L})=s$. Let $g_{d}^{n}$ and $R$ be in the Setting up. If $g_{d}^{n}$ is birationally very ample with $n \geq 3$ and $g>\max \{\pi(a-2 s+2+2 r, r), 6(a-2 s)+9\}$ for some $r \leq n$, then $C$ is trigonal and

$$
\mathcal{L} \simeq \mathcal{O}_{C}\left(l g_{3}^{1}+R\right),\left|l g_{3}^{1}\right|=g_{3 l}^{l}, \quad l=\frac{2 g-2-d}{3} \geq \frac{g-1}{6}
$$

Proof. Suppose $d \leq g-1$. Then $d \geq 3 n-2$ using Castelnuovo's genus bound. Then we can use Castelnuovo number's property $\pi(d-2, r-1) \geq \pi(d, r)$ in Lemma 6 in [8], and so $\operatorname{Cliff}\left(g_{d}^{n}\right) \leq a-2 s+2$ yields that

$$
g \leq \pi(d, n) \leq \pi(d-2 n+2 r, r) \leq \pi(a-2 s+2+2 r, r)
$$

for $r \leq n$, which cannot occur by the genus condition.

If $\bar{d} \geq g$, then $n \leq \frac{2 d-g+1}{3}$ by Castelnuovo's genus bound. If $n \leq \frac{2 d-g}{3}$, then

$$
\begin{aligned}
a-2 s-b+2 & \geq \operatorname{Cliff}\left(\mathcal{K} \otimes \mathcal{A}^{-1}\right)-b=\operatorname{Cliff}\left(g_{d}^{n}\right) \\
& \geq \frac{(2 g-2-d)+2}{3}=\frac{\operatorname{deg} \mathcal{A}+2}{3} \\
& \geq \frac{g+3}{6}
\end{aligned}
$$

which is absurd. Thus we have $n=\frac{2 d-g+1}{3}$ and $C$ is an extremal curve. Note that $C$ is nonhyperelliptic since $C$ has a special birationally very ample $g_{d}^{n}$. By (2.6) Corollary in Chapter III of [1], $C$ is a trigonal curve and $g_{d}^{n}=$ $\left|\mathcal{K} \otimes \mathcal{A}^{-1}\right|=\left|\mathcal{K}\left(-l g_{3}^{1}\right)\right|,\left|l g_{3}^{1}\right|=g_{3 l}^{l}, l=\frac{2 g-2-d}{3}$. Thus we have $\mathcal{L} \simeq \mathcal{O}_{C}\left(l g_{3}^{1}+R\right)$ since $\mathcal{A} \simeq \mathcal{L}(-R)$.

Remark 2.4. For the very ampleness of a nonspecial line bundle $\mathcal{L} \simeq \mathcal{K}-g_{d}^{n}-$ $B_{b}+R$, we need to have the condition $\operatorname{deg} R \geq n+3$.

Also we have known the following on the normal generation of nonspecial line bundles on trigonal curves or double coverings of a curve. For the later use we state the following propositions.

Proposition 2.5 (Martens and Schreyer [12], Corollary 1). Let $C$ be a trigonal curve of genus $g>4$. Let $\mathcal{L}$ be a nonspecial very ample line bundle of degree $2 g-k$ with $0 \leq k \leq M$ where $M$ is the Maroni invariant of the curve $C$. Then $\mathcal{L}$ is not normally generated if and only if $\mathcal{L}$ is isomorphic to $\mathcal{O}\left(\mathcal{K}-k g_{3}^{1}+D_{2 k+2}\right)$ for some effective divisor $D_{2 k+2} \in C_{2 k+2}$.

Proposition 2.6 (Lange and Martens [11]). Let $C$ be a double covering of genus $g$ of a smooth curve $C^{\prime}$ of genus $p$. Let $j$ be an integer such that $3 p+1 \leq$ $j \leq g-4$ if $p \geq 2,4 \leq j \leq g-3$ if $p=1$ and $j \geq 1$ if $p=0$. Then $C$ admits no nonspecial normally generated line bundle of degree $2 g+1-j$. 


\section{Main results}

In this work we deal with the normal generation of nonspecial line bundles of degree $\leq 2 g-2$. Recall that the normal generation of nonspecial line bundles $\mathcal{L}$ of $\operatorname{deg} \mathcal{L} \geq 2 g-5$ has known by several authors. Here, using Lemma 2.3, we get our main theorem. As an application of the theorem, we obtain a new result on the normal generation of nonspecial line bundles $\mathcal{L}$ with $\operatorname{deg} \mathcal{L} \leq 2 g-6$ and previous results with $2 g-5 \leq \operatorname{deg} \mathcal{L} \leq 2 g-2$.

Theorem 3.1. Let $C$ be a smooth curve of genus $g$ and $\mathcal{L}$ a nonspecial very ample line bundle with $\operatorname{deg} \mathcal{L}=2 g-2-a>\frac{3 g+1}{2}$. If $\mathcal{L}$ fails to be normally generated, then

$$
\mathcal{L} \simeq \mathcal{K}-g_{d}^{n}-B_{b}+R_{d+b-a} \simeq \mathcal{K}-g_{l}^{0}-B_{b}+R
$$

$\operatorname{Supp}\left(g_{l}^{0}+B_{b}\right) \cap \operatorname{Supp}(R)=\emptyset, \operatorname{deg} R \geq 3,3+a+n \leq d+b \leq 2+a+2 n$,

for a base point free complete $g_{d}^{n}$ and a complete $g_{l}^{0} \leq g_{d}^{n}$ with $B_{b} \in C_{b}, R \leq$ $R_{d+b-a} \in C_{d+b-a}$, whose precise form is one of the following;

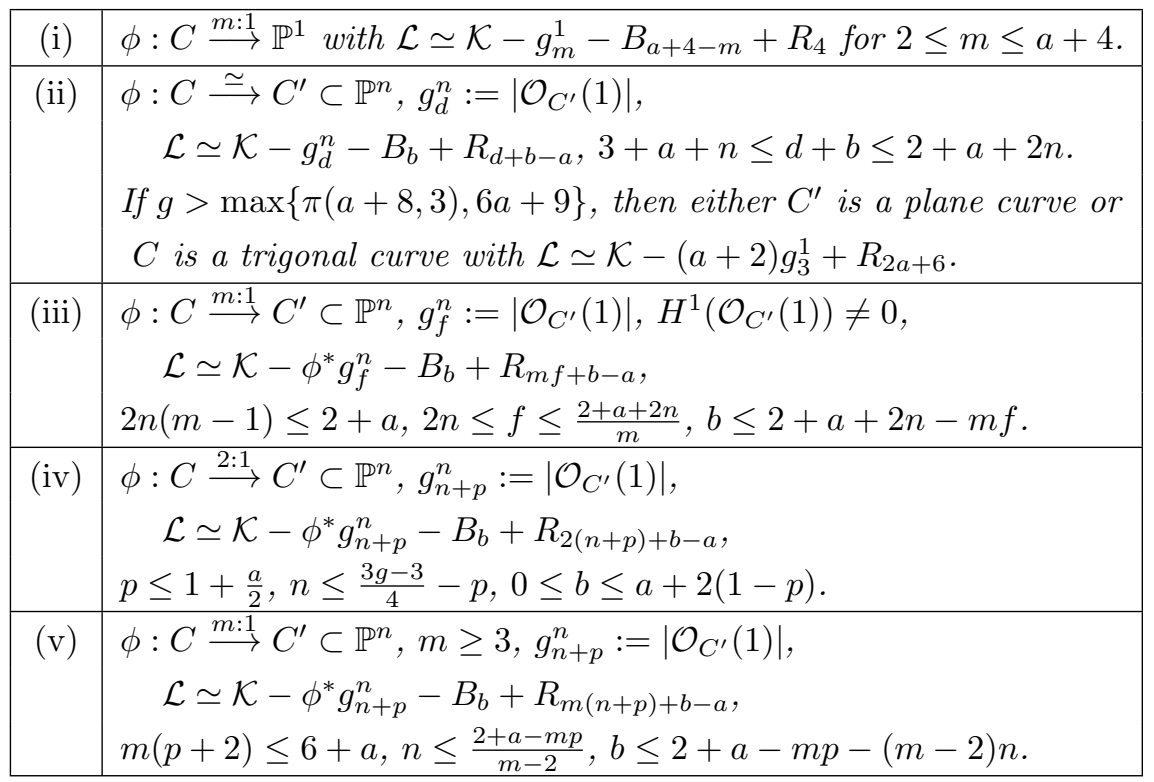

Here, $\phi$ is the morphism associated to $g_{d}^{n}$ and $p$ is the genus of $C^{\prime}$ and $n \geq 2$ in (ii)-(v). For the convenience we identify $C^{\prime}$ with the smooth model of $C^{\prime}$.

Proof. Assume $\mathcal{L}$ is not normally generated, then by the Setting up in Section 2

$$
\mathcal{L} \simeq \mathcal{K}-g_{d}^{n}-B_{b}+R_{d+b-a}
$$

for a base point free complete $g_{d}^{n}$ and some $B_{b} \in C_{b}, R_{d+b-a} \in C_{d+b-a}$. Note that

$$
\mathcal{L} \simeq \mathcal{K}-g_{d}^{n}-B_{b}+R_{d+b-a} \simeq \mathcal{K}-g_{l}^{0}-B_{b}+R
$$


for some $g_{l}^{0} \leq g_{d}^{n}$ with $h^{0}\left(C, g_{l}^{0}\right)=1$, and some $R \leq R_{d+b-a}$ with $\operatorname{deg} R \leq$ $d+b-a-n, \operatorname{Supp}\left(g_{l}^{0}+B_{b}\right) \cap \operatorname{Supp}(R)=\emptyset$. By the very ampleness of $\mathcal{L}$, we have $\operatorname{deg} R \geq 3$, and hence $d+b-a-n \geq 3$. Also we obtain $d \leq 2+a+2 n-b$ from Cliff $\left(g_{d}^{n}\right) \leq 2+a-b$. Therefore we have $3+a+n \leq d+b \leq 2+a+2 n$.

Let $\phi$ be the morphism associated with $g_{d}^{n}$ and $C^{\prime}$ the image curve $\phi(C)$. For the convenience, we identify $C^{\prime}$ with the smooth model of $C^{\prime}$. Then we have $g_{d}^{n}=\left|\phi^{*} \mathcal{O}_{C^{\prime}}(1)\right|$.

If $n=1$, then $g_{d}^{1}=g_{m}^{1}$ and $\mathcal{L} \simeq \mathcal{K}-g_{m}^{1}-B_{a+4-m}+R_{4}$ for $m \leq a+4$ by Remark 2.1. Thus we get (i). Therefore we may assume $n \geq 2$.

If $g_{d}^{n}$ is birationally very ample, then $C$ is birationally isomorphic to $C^{\prime}$ of degree $d$ in $\mathbb{P}^{n}$. If we assume $g>\max \{\pi(a+8,3), 6 a+9\}$, then $n=2$ or $C$ is a trigonal curve by Lemma 2.3. Moreover, by Proposition 2.5 we have $\mathcal{L} \simeq \mathcal{K}-(a+2) g_{3}^{1}+R_{2 a+6}$ since the Maroni invariant of $C$ is no less than $a+2$. Thus we get (ii).

Suppose $g_{d}^{n}$ is not birationally very ample. Consider the morphism

$$
\phi: C \rightarrow C^{\prime} \subset \mathbb{P}^{n} .
$$

Set $m:=\operatorname{deg} \phi \geq 2, p:=g\left(C^{\prime}\right)$. Then $\left|\mathcal{O}_{C^{\prime}}(1)\right|=g_{\frac{d}{m}}^{n}, g_{d}^{n}=\phi^{*} g_{\frac{d}{m}}^{n}$, and

$$
\mathcal{L} \simeq \mathcal{K}-\phi^{*} g_{\frac{d}{m}}^{n}-B_{b}+R_{d+b-a} .
$$

If $H^{1}\left(C^{\prime}, \mathcal{O}_{C^{\prime}}(1)\right) \neq 0$, then by Clifford's theorem and $\operatorname{Cliff}\left(g_{d}^{n}\right) \leq 2+a-b$

$$
2 n \leq \frac{d}{m} \leq \frac{2+a-b+2 n}{m} \leq \frac{2+a+2 n}{m} .
$$

Accordingly $2 n(m-1) \leq 2+a, \quad 0 \leq b \leq 2+a+2 n-\frac{d}{m} \cdot m$. Thus we have (iii).

Assume $H^{1}\left(C^{\prime}, \mathcal{O}_{C^{\prime}}(1)\right)=0$, then $\left|\mathcal{O}_{C^{\prime}}(1)\right|=g_{n+p}^{n}, n=\frac{d}{m}-p$ and

$$
\frac{(m-2) d}{m}+2 p=\operatorname{Cliff}\left(g_{d}^{n}\right) \leq 2+a-b .
$$

Therefore

$$
(m-2)(p+2) \leq(m-2)(p+n)=(m-2) \frac{d}{m} \leq 2+a-b-2 p .
$$

If $m=2$, then $p \leq 1+\frac{a}{2}, 0 \leq b \leq a+2(1-p)$ and

$$
\mathcal{L} \simeq \mathcal{K}-\phi^{*} g_{n+p}^{n}-B_{b}+R_{2(n+p)+b-a} .
$$

Here, $2 \leq n \leq \frac{3 g-3}{4}-p$, since $\operatorname{deg} \mathcal{A} \geq \frac{g-1}{2}$ and $d \leq 2 g-2-\operatorname{deg} \mathcal{A}$. Hence we get (iv).

If $m \geq 3$, then $m(p+2) \leq 6+a, n \leq \frac{2+a-m p}{m-2}, 0 \leq b \leq 2+a-m p-(m-2) n$, and

$$
\mathcal{L} \simeq \mathcal{K}-\phi^{*} g_{n+p}^{n}-B_{b}+R_{m(n+p)+b-a} .
$$

Therefore we obtain (v). In all, we obtain the results. 
Remark 3.2. (1) In Theorem 3.1, the line bundle $\mathcal{L} \simeq \mathcal{K}-g_{d}^{n}-B_{b}+R_{d+b-a}$ can have another representation as follows. Consider $g_{d}^{n}\left(-R_{k}\right)$ for some $R_{k} \leq$ $R_{d+b-a}, \operatorname{deg} R_{k}=k \leq n$. Then $g_{d}^{n}\left(-R_{k}\right)=g_{d^{\prime}}^{n^{\prime}}+B_{d^{\prime}}, n^{\prime} \geq n-k \geq 0$, where $g_{d^{\prime}}^{n^{\prime}}$ is base point free and $B_{d^{\prime}}$ is the base locus of $g_{d}^{n}\left(-R_{k}\right)$. Consequently $\mathcal{L}$ can be also written by $\mathcal{L} \sim \mathcal{K}-g_{d^{\prime}}^{n^{\prime}}-\left(B_{b^{\prime}}+B_{b}\right)+\left(R_{d+b-a}-R_{k}\right)$.

(2) For $m=3$ in Theorem 3.1 (i), we have the following:

$$
\begin{aligned}
\mathcal{K}-g_{3}^{1}-B_{a+1}+R_{4} & \simeq \mathcal{K}-g_{3}^{1}-\left(B_{a+1}+g_{2 a+2}^{0}\right)+g_{2 a+2}^{0}+R_{4} \\
& \simeq \mathcal{K}-(a+2) g_{3}^{1}+D_{2 a+6}, D_{2 a+6}:=g_{2 a+2}^{0}+R_{4},
\end{aligned}
$$

where we choose $g_{2 a+2}^{0}$ to be $\left|B_{a+1}+g_{2 a+2}^{0}\right|=\left|(a+1) g_{3}^{1}\right|$ (such a choice is always possible). Consequently, this case is collapsed with Theorem 3.1(iii). Thus the converse of the case $m=3$ in Theorem 3.1(i) with $a \leq \frac{g-10}{3}$ also holds by Proposition 2.5.

From Theorem 3.1, we have the following:

Corollary 3.3. Let $C$ be a smooth curve of genus $g>33$ and $\mathcal{L}$ a nonspecial very ample line bundle with $\operatorname{deg} \mathcal{L}=2 g-6$. If $\mathcal{L}$ fails to be normally generated, then $(C, \mathcal{L})$ falls into one of following cases;

\begin{tabular}{|c|l|}
\hline (i) & $\phi: C \stackrel{m: 1}{\rightarrow} \mathbb{P}^{1}$ with $\mathcal{L} \simeq \mathcal{K}-g_{m}^{1}-B_{8-m}+R_{4}$ for $2 \leq m \leq 8$. \\
\hline (ii) & $C$ is either a plane curve of degree 10 with $\mathcal{L} \simeq \mathcal{K}-g_{10}^{2}+R_{6}$, \\
& or a trigonal curve with $\mathcal{L} \simeq \mathcal{K}-6 g_{3}^{1}+R_{14}$.
\end{tabular}

This box corresponds to the one in Theorem 3.1.

Proof. If we plug $a=4$ in the result of Theorem 3.1, then we get the following:

(i) $C$ is a $m$-fold covering of $\mathbb{P}^{1}$ and $\mathcal{L} \simeq \mathcal{K}-g_{m}^{1}-B_{8-m}+R_{4}$ for $m \leq 8$. 
(ii) Since $g>6 a+9=33>\pi(a+8,3)=25, C$ is birational to a curve $C^{\prime} \subset \mathbb{P}^{2}$ of degree 10 and $\mathcal{L} \simeq \mathcal{K}-g_{10}^{2}+R_{6}$ or $C$ is a trigonal curve and $\mathcal{L} \simeq \mathcal{K}-6 g_{3}^{1}+R_{14}$.

(iii) From the condition $2 n(m-1) \leq 2+a$, we have $m=2$ and $2 \leq n \leq 3$, which yields $4 \leq f \leq 5$ for $n=2$ and $f=6$ for $n=3$, since $2 n \leq f \leq \frac{6+2 n}{2}$. In particular, if $n=2$ and $f=4$, then $C^{\prime}$ is smooth since $H^{1}\left(C^{\prime}, \overline{\mathcal{O}}_{C^{\prime}}(1)\right) \neq 0$. Assume $n=3, f=6$, then $p \leq 4$ by Castelnuovo genus bound. Note that $\operatorname{deg} C^{\prime} \leq 2 g\left(C^{\prime}\right)-2$ for $H^{1}\left(C^{\prime}, \mathcal{O}_{C^{\prime}}(1)\right) \neq 0$, whence $6=\operatorname{deg} C^{\prime} \leq 2 p-2$ and then $p \geq 4$. Therefore $p=4$ and $\operatorname{deg} C^{\prime}=2 p-2=6$ with $H^{1}\left(C^{\prime}, \mathcal{O}_{C^{\prime}}(1)\right) \neq 0$, and so $\mathcal{O}_{C^{\prime}}(1) \simeq K_{C^{\prime}}$.

(iv) $C$ is a double covering of a curve $C^{\prime} \subset \mathbb{P}^{n}$ of genus $p \leq 3$ with $H^{1}\left(C^{\prime}, \mathcal{O}_{C^{\prime}}(1)\right)=0$ and $\mathcal{L} \simeq \mathcal{K}-\phi^{*} g_{n+p}^{n}-B_{b}+R_{2(n+p)-a+b}$ for $2 \leq n \leq \frac{3 g-3}{4}-p$ and $b \leq 6-2 p$.

(v) $C$ is a $m$-fold covering of $C^{\prime} \subset \mathbb{P}^{n}$ such that $H^{1}\left(C^{\prime}, \mathcal{O}_{C^{\prime}}(1)\right)=0, \mathcal{L} \simeq$ $\mathcal{K}-\phi^{*} g_{n+p}^{n}-B_{b}+R_{m(n+p)+b-4}, 3 \leq m \leq 5,0 \leq p \leq \frac{10}{m}-2, n \leq \frac{6-m p}{m-2}$, $b \leq 6-m p-(m-2) n$.

Consider the case for $m=3$. First if $p=0$, in other words, $C$ is a trigonal curve, then by Proposition 2.5 we get $\mathcal{L} \simeq \mathcal{K}-6 g_{3}^{1}+R_{14}$ since the Maroni invariant of $C$ is no less than $\frac{g-4}{3}$. If $p=1$, then $n=2,3, b \leq 3-n$, and we have $\mathcal{L} \simeq \mathcal{K}-\phi^{*} g_{n+1}^{n}-B_{b}+R_{3(n+1)+b-4}$.

If $m=4$, then we obtain $p=0, n=2,3, b \leq 6-2 n$, and $\mathcal{L} \simeq \mathcal{K}-\phi^{*} g_{n}^{n}-$ $B_{b}+R_{4 n+b-4}$.

If $m=5$, then we have $p=0, n=2, b=0$, and $\mathcal{L} \simeq \mathcal{K}-\phi^{*} g_{2}^{2}+R_{6}$.

For the cases with $0 \leq a \leq 3$, we have the following remarks.

Remark 3.4. For the case $a=0$ in Theorem 3.1 with $g>9$ in this paper we have:

\begin{tabular}{|c|l|}
\hline (i) & $\phi: C \stackrel{m: 1}{\rightarrow} \mathbb{P}^{1}$ with $\mathcal{L} \simeq \mathcal{K}-g_{m}^{1}-B_{4-m}+R_{4}$ for $2 \leq m \leq 4$. \\
\hline (ii) & $C$ is a smooth plane curve of degree 6 with $\mathcal{L} \simeq \mathcal{K}-g_{6}^{2}+R_{6}$, \\
& or $C$ is a trigonal curve with $\mathcal{L} \simeq \mathcal{K}-2 g_{3}^{1}+R_{6}$. \\
\hline (iii) & This case cannot happen since $4(m-1) \leq 2 n(m-1) \leq 2$ is impossible. \\
\hline (iv) & $\phi: C \stackrel{2: 1}{\longrightarrow} C^{\prime} \subset \mathbb{P}^{n}, 0 \leq p \leq 1, \mathcal{L} \simeq \mathcal{K}-\phi^{*} g_{n+p}^{n}-B_{b}+R_{2(n+p)+b}$, \\
& $2 \leq n \leq \frac{3 g-3}{4}-p, b \leq 2-2 p$. \\
\hline (v) & $C$ is a trigonal curve and $\mathcal{L} \simeq \mathcal{K}-2 g_{3}^{1}+R_{6}$ by Proposition 2.5. \\
\hline
\end{tabular}

This box also corresponds to the one in Theorem 3.1.

Comparing this result to:

Proposition 3.5 ([10], Theorem 3.1). Let $X$ be a smooth irreducible projective algebraic curve of genus $g \geq 15$. Let $\mathcal{L}$ be a very ample nonspecial line bundle of degree $2 g-2$. Then $\mathcal{L}$ fails to be normally generated if and only if either: 
(a) $X$ is hyperelliptic;

(b) $X$ is bielliptic and $\mathcal{L} \simeq \mathcal{K}-\pi^{*} g_{2}^{1}+D_{4}$ for some $D_{4} \in X_{4}$;

(c) $X$ is bielliptic and $\mathcal{L} \simeq \mathcal{K}-\pi^{*} g_{n+1}^{n}+D_{2 n+2}, \pi_{*}\left(D_{2 n+2}\right) \not 2 g_{n+1}^{n}$ for some $2 \leq n \leq g-6$ and $D_{2 n+2} \in X_{2 n+2}$;

(d) $X$ is trigonal and $\mathcal{L} \simeq \mathcal{K}-2 g_{3}^{1}+D_{6}$ for some $D_{6} \in X_{6}$ or

(e) $X$ is simple 4-gonal and $\mathcal{L} \simeq \mathcal{K}-g_{4}^{1}+D_{4}$ for some $D_{4} \in X_{4}$.

Then we see the similarities except case (ii); the plane curve case in (ii) does not appear in Proposition 3.5 since they assumed $g \geq 15>\pi(6,2)$. Also, we can easily see that the converse holds by the existence of four collinear points or Proposition 2.5 or Proposition 2.6.

Remark 3.6. For the case $a=1$ in Theorem 3.1 with $g>15$ in this paper we have

\begin{tabular}{|c|l|}
\hline (i) & $\phi: C \stackrel{m: 1}{\rightarrow} \mathbb{P}^{1}$ with $\mathcal{L} \simeq \mathcal{K}-g_{m}^{1}-B_{5-m}+R_{4}$ for $2 \leq m \leq 5$. \\
\hline (ii) & $C$ is a trigonal curve with $\mathcal{L} \simeq \mathcal{K}-3 g_{3}^{1}+R_{8}$. \\
\hline (iii) & This case cannot occur since $4(m-1) \leq 2 n(m-1) \leq 3$ is absurd. \\
\hline (iv) & $\phi: C \stackrel{2: 1}{\longrightarrow} C^{\prime} \subset \mathbb{P}^{n}, 0 \leq p \leq 1, \mathcal{L} \simeq \mathcal{K}-\phi^{*} g_{n+p}^{n}-B_{b}+R_{2(n+p)-1+b}$, \\
& $2 \leq n \leq \frac{3 g-3}{4}-p, b \leq 3-2 p$. \\
\hline (v) & $C$ is a trigonal curve with $\mathcal{L} \simeq \mathcal{K}-3 g_{3}^{1}+R_{8}$ by Proposition 2.5. \\
\hline
\end{tabular}

This box also corresponds to the one in Theorem 3.1.

Comparing this result to:

Proposition 3.7 ([10], Theorem 3.2). Let $X$ be a smooth irreducible projective algebraic curve of genus $g \geq 17$. Let $\mathcal{L}$ be a very ample nonspecial line bundle of degree $2 g-3$. Then $\mathcal{L}$ fails to be normally generated if and only if either:
(a) $X$ is hyperelliptic;
(b) $X$ is bielliptic and $\mathcal{L} \simeq \mathcal{K}-\pi^{*} g_{n+2}^{n+1}+D_{2 n+3}$ for some $1 \leq n \leq g-7$;
(c) $X$ is trigonal and $\mathcal{L} \simeq \mathcal{K}-3 g_{3}^{1}+D_{8}$ for some $D_{8} \in X_{8}$;
(d) $X$ is simple 4-gonal and $\mathcal{L} \simeq \mathcal{K}-g_{4}^{1}+D_{3}$ for some $D_{3} \in X_{3}$ or
(e) $X$ is 5-gonal and $\mathcal{L} \simeq \mathcal{K}-g_{5}^{1}+D_{4}$ for some $D_{4} \in X_{4}$.

The case (d) in Proposition 3.7 does not appear at the box of Remark 3.6 since $h^{0}(C, \mathcal{L})-h^{0}\left(\mathcal{L}\left(-D_{3}\right)\right)=1$ and so $\mathcal{L}$ is not very ample. Also, we can see that the converse for the other cases holds by the existence of four collinear points or Proposition 2.5 or Proposition 2.6.

Remark 3.8. For the cases $a=2,3$ in Theorem 3.1 with $g>6 a+9$ in this paper we have the followings: 


\begin{tabular}{|c|c|}
\hline (i) & $\phi: C \stackrel{m: 1}{\longrightarrow} \mathbb{P}^{1}$ with $\mathcal{L} \simeq \mathcal{K}-g_{m}^{1}-B_{a+4-m}+R_{4}$ for $2 \leq m \leq a+4$ \\
\hline (ii) & $\begin{array}{l}C \text { is a plane curve } C^{\prime} \text { of degree } 9 \text { with } \mathcal{L} \simeq \mathcal{K}-g_{9}^{2}+R_{6} \text { with } a=3 \text {. } \\
\text { or } C \text { is a trigonal curve with } \mathcal{L} \simeq \mathcal{K}-(a+2) g_{3}^{1}+R_{2 a+6} .\end{array}$ \\
\hline (iii) & $\begin{array}{l}\text { Since } 2 n(m-1) \leq a+2, \text { we get } m=2, n=2 . \\
C \text { is a double covering of smooth plane quartic curve } C^{\prime} \\
\mathcal{L} \simeq \mathcal{K}-\phi^{*} g_{4}^{2}-B_{b}+R_{8+b-a}, 0 \leq b \leq a-2\end{array}$ \\
\hline (iv) & $\begin{array}{l}\phi: C \stackrel{2: 1}{\longrightarrow} C^{\prime} \subset \mathbb{P}^{n}, 0 \leq p \leq 2, \mathcal{L} \simeq \mathcal{K}-\phi^{*} g_{n+p}^{n}-B_{b}+R_{2(n+p)-a+b}, \\
2 \leq n \leq \frac{3 g-3}{4}-p, b \leq 2+a-2 p .\end{array}$ \\
\hline$(\mathrm{v})$ & $\begin{aligned} & \phi: C \stackrel{m: 1}{\longrightarrow} C^{\prime} \subset \mathbb{P}^{n}, m \geq 3, m(p+2) \leq 6+a, \\
& \mathcal{L} \simeq \mathcal{K}-\phi^{*} g_{n+p}^{n}-B_{b}+R_{m(n+p)+b-a}, \\
& n \leq \frac{2+a-m p}{m-2} \text { and } b \leq 2+a-m p-(m-2) n . \\
& \text { Consequently, } C \text { is one of the followings; } \\
& \phi: C \stackrel{3: 1}{\rightarrow} \mathbb{P}^{1}, \mathcal{L} \simeq \mathcal{K}-(2+a) g_{3}^{1}+R_{2 a+6} \text { by Proposition } 2.5, \\
& \phi: C \stackrel{3: 1}{\rightarrow} C^{\prime}, g\left(C^{\prime}\right)=1, a=3, \text { and } \mathcal{L} \cong \mathcal{K}-\phi^{*} g_{3}^{2}+R_{6}, \\
& \phi: C \stackrel{4: 1}{\rightarrow} \mathbb{P}^{1} \text { and } \mathcal{L} \cong \mathcal{K}-\phi^{*} g_{2}^{2}-B_{b}+R_{8+b-a}, b \leq a-2 .\end{aligned}$ \\
\hline
\end{tabular}

This box also corresponds to the one in Theorem 3.1.

This result is exactly same as Proposition 3.9 except case (ii). In fact, the plane curve case in (ii) does not appear in Proposition 3.9, since they assumed $g>\frac{(4+a)(5+a)}{2}$.

Proposition 3.9 ([9], Theorem 1.1). Let $C$ be a smooth curve of genus $g$ and $L$ be a nonspecial very ample line bundle on $C$ with $\operatorname{deg} L=2 g-2-a, a=2,3$ and $g>\frac{(4+a)(5+a)}{2}$. If $L$ fails to be normally generated, then either

(a) $C$ is a double covering of a genus $p$ curve $C^{\prime}, 0 \leq p \leq 2$ and $\mathcal{L} \cong \mathcal{K}-\phi^{*} g_{n+p}^{n}-B_{b}+R_{2(n+p)-a+b}, 0 \leq b \leq a+2-2 p, 2 \leq n \leq \frac{3 g-3}{4}-p ;$

(b) $C$ is trigonal and $\mathcal{L} \cong \mathcal{K}-(2+a) g_{3}^{1}+R_{2 a+6}$;

(c) $C$ is a triple covering of an elliptic curve $C^{\prime}$ and $a=3, \mathcal{L} \cong \mathcal{K}-\phi^{*} g_{3}^{2}+R_{6}$;

(d) $C$ is 4-gonal and $\mathcal{L} \cong \mathcal{K}-2 g_{4}^{1}-B_{b}+R_{8-a+b}, 0 \leq b \leq a-2$;

(e) $C$ has a $g_{4+a}^{1}$ and $\mathcal{L} \cong \mathcal{K}-g_{4+a}^{1}+R_{4}$ or

(f) $C$ is a double covering of a smooth plane quartic curve $C^{\prime}$ and

$$
\mathcal{L} \cong \mathcal{K}-\phi^{*} g_{4}^{2}-B_{b}+R_{8-a+b}, \quad 0 \leq b \leq a-2 .
$$

Here we mean that $B_{b} \in C_{b}, R_{i} \in C_{i}$ and $\phi$ is a covering morphism from $C$ to $C^{\prime}$. The converse holds for (a) with $p=0,1$, (b) and (e).

\section{References}

[1] E. Arbarello, M. Cornalba, P. A. Griffiths, and J. Harris, Geometry of Algebraic Curves. Vol. I, Springer-Verlag, New York, 1985.

[2] E. Ballico, On the Clifford index of algebraic curves, Proc. Amer. Math. Soc. 97 (1986), no. $2,217-218$. 
[3] G. Castelnuovo, Sui multipli di una serie lineare di gruppi di punti appartenente ad una curva algebrica, Rend. Circ. Mat. Palermo 7 (1893), 89-110.

[4] M. Coppens and G. Martens, Secant spaces and Clifford's theorem, Compositio Math. 78 (1991), no. 2, 193-212.

[5] M. Green and R. Lazarsfeld, On the projective normality of complete linear series on an algebraic curve, Invent. Math. 83 (1985), no. 1, 73-90.

[6] R. Hartshorne, Algebraic Geometry, Graduate Text in Math, 52, Berlin-Heidelberg-New York 1977.

[7] C. Keem and S. Kim, On the Clifford index of a general (e+2)-gonal curve, Manuscripta Math. 63 (1989), no. 1, 83-88.

[8] S. Kim and Y. Kim, Projectively normal embedding of a k-gonal curve, Comm. Algebra 32 (2004), no. 1, 187-201.

[9] , Normal generation of line bundles on algebraic curves, J. Pure Appl. Algebra 192 (2004), no. 1-3, 173-186.

[10] T. Kato, C. Keem, and A. Ohbuchi, Normal generation of line bundles of high degrees on smooth algebraic curves, Abh. Math. Sem. Univ. Hamburg 69 (1999), 319-333.

[11] H. Lange and G. Martens, Normal generation and presentation of line bundles of low degree on curves, J. Reine Angew. Math. 356 (1985), 1-18.

[12] G. Martens and F. O. Schreyer, Line bundles and syzygies of trigonal curves, Abh. Math. Sem. Univ. Hamburg 56 (1986), 169-189.

[13] D. Mumford, Varieties defined by quadric equations, Questions on Algebraic Varieties (C.I.M.E., III Ciclo, Varenna, 1969) pp. 29-100 Edizioni Cremonese, Rome, 1970.

SEONJA Kim

Department of Electronics

Chungwoon University

Chungnam 350-701, Korea

E-mail address: sjkim@chungwoon.ac.kr

YOUNG Rock KIM

Department of Mathematics Education

Graduate School of Education

Hankuk University of Foreign Studies

SeOul 130-791, Korea

E-mail address: rocky777@hufs.ac.kr 